\title{
Augmented Secure Channels and the Goal of the TLS 1.3 Record Layer
}

\author{
Christian Badertscher ${ }^{1}$, Christian Matt ${ }^{1}$, Ueli Maurer ${ }^{1}$, \\ Phillip Rogaway ${ }^{2}$, and Björn Tackmann ${ }^{3}$ \\ 1 Department of Computer Science, ETH Zurich, Switzerland \\ $\{$ badi, mattc, maurer\}@inf.ethz.ch \\ 2 Department of Computer Science, University of California, Davis, USA \\ rogaway@cs.ucdavis.edu \\ 3 Department of Computer Science \& Engineering, \\ University of California, San Diego, USA \\ btackmann@eng.ucsd.edu
}

\begin{abstract}
Motivated by the wide adoption of authenticated encryption and TLS, we suggest a basic channel abstraction, an augmented secure channel (ASC), that allows a sender to send a receiver messages consisting of two parts, where one is privacy-protected and both are authenticityprotected. Working in the tradition of constructive cryptography, we formalize this idea and provide a construction of this kind of channel using the lower-level tool authenticated-encryption.

We look at recent proposals on TLS 1.3 and suggest that the criterion by which their security can be judged is quite simple: do they construct an ASC? Due to this precisely defined goal, we are able to give a natural construction that comes with a rigorous security proof and directly leads to a proposal on TLS 1.3 that is provably secure.
\end{abstract}

\section{Introduction}

This paper defines and investigates a new abstraction of a secure channel. We call it an augmented secure channel, or ASC. Like most types of channels, an ASC lets a sender Alice send messages to a receiver Bob. But unlike more conventional types of channels, each message has designated private and non-private parts. An active adversary Eve occupies the system, but is limited to seeing the length of the private portion and the contents of the non-private portion of each message - and to entirely shutting down the channel. In particular, the adversary cannot inject messages or induce out-of-order message delivery. Additionally, the non-private portion can contain an implicit part, already known to the receiver, that is not transmitted but still authenticated, e.g., to bind the message to a given context.

The service an ASC provides is motivated by the ascendancy of both TLS and authenticated encryption. We take the rise of these tools, and what they

\footnotetext{
* This is the full version of a paper due to appear at the 9th International Conference on Provable Security (ProvSec 2015). The final publication will be available at link. springer. com.
} 
deliver, as an indication that customary conceptualizations of secure channels may not have been rich enough to deliver the service that protocol designers routinely need.

Authenticated encryption. While ASCs are closely related to schemes for authenticated encryption (AE) and authenticated encryption with associated data (AEAD), an ASC and an AE/AEAD-scheme are very different things. An ASC is a reasonably high-level object: an abstract resource that parties can employ, getting compositional guarantees when they do. Our formulation of ASCs will be in the tradition of constructive cryptography [16 17]. In contrast, an AEAD-scheme is a comparatively low-level primitive: it is a tuple of algorithms that is "good" in some particular, complexity-theoretic sense.

The AEAD notion emerged over a sequence of works [2]3]12]13|22|23|24] having two distinct purposes: to minimize the misuse of symmetric encryption primitives and to gain efficiency advantages over generic composition schemes (i.e., traditional ways to meld privacy-only encryption schemes and messageauthentication codes). But in moving from conventional encryption to AEAD, the basic conception of what symmetric encryption $i s$ was thoroughly revamped: authenticity became an intrinsic part of the goal; so too did the allowance of (nonprivate) associated data $A$; while probabilism, formerly seen as indispensable, was surfaced and subsumed by a nonce $N$. Roughly said, an AEAD-scheme would nowadays be defined as a triple of algorithms $\Pi=(\mathcal{K}, \mathcal{E}, \mathcal{D})$ where a computationally-reasonable adversary $\mathcal{A}$ has poor advantage at distinguishing encryption and decryption oracles $\left(\mathcal{E}_{K}(N, A, M), \mathcal{D}_{K}(N, A, C)\right)$ from a pair of oracles $(\$(N, A, M), \perp(N, A, C))$, where $K$ is generated by $\mathcal{K}$, the $\$(N, A, M)$ oracle returns an appropriate number of random bits, the $\perp(N, A, C)$ oracle always returns $\perp$, the adversary repeats no nonce $N$ in queries to its first oracle, and queries that would result in trivial wins are disallowed.

The new conceptualization for symmetric encryption gained surprisingly rapid acceptance. The IEEE, IETF, ISO, and NIST all stepped in to standardize AEAD-schemes (e.g., in NIST SP 800-38C and SP 800-38D, IEEE 802.11i, ISO 19772, and IETF RFC 3610, 5116, 5288, 5297, and 7253). Methods that had been previously embedded in widely-deployed systems and standards, (e.g., SSH, and SSL) were recognized as attempts - sometimes rather clumsy ones - to achieve $\mathrm{AE} / \mathrm{AEAD}$. Revisions to widely-used protocols started to deploy the ready-built solutions to AEAD rather than the ad hoc and error-prone mechanisms that had provided no real abstraction boundaries other that of block ciphers, hash functions, or MACs.

Understanding the goal of TLS. A long line of work analyzes the security of TLS (mainly versions prior to 1.3) [8911/14]15|19|2025]. Several recent papers [11[15] use a security notion called Authenticated and Confidential Channel Establishment (ACCE), a game-based definition that models both the handshake and the record layer, as TLS versions prior to 1.3 could formally not be proved as the composition of the two sub-protocols. Motivated by the adoption of AEAD 
as well as the better separation of the two sub-protocols in TLS 1.3, we give a novel interpretation for the goal of the TLS record layer: constructing a specific instantiation of an ASC, from insecure communication and a shared secret key constructed by the handshake sub-protocol. Indeed, messages in the TLS record protocol consist of private and non-private parts, which are both authenticated.

We show how a generic ASC construction directly leads to this specific instantiation of an ASC. We thereby obtain a provably secure TLS record protocol. Our proposal differs from the current draft for TLS 1.3 by slightly reducing the size of transmitted records and the number of elements in the authenticated data, as well as a different choice of the nonces.

The gap between a scheme's security properties and its use. Classical cryptographic definitions, including the AEAD definition reviewed above, do not capture in which contexts a scheme satisfying them can securely be used. They consider a specific attack model and give certain capabilities to an adversary that tries to win some game, but it is not a priori clear which capabilities an adversary has in a particular application, or even what her final goal is. To illustrate our point, consider the standard notions for encryption schemes, IND-CPA and IND-CCA. While IND-CCA is stronger, it is not obvious in which applications an IND-CCA encryption scheme is needed and where IND-CPA would suffice. These considerations are highly security-relevant. For complex protocols like TLS or IPSec, one has to make sure that any overall attack can be translated to an attack against the CPA or CCA game or another hardness assumption; only then the protocol is sound. But such analyses are complex and cannot be reused for the analysis of other protocols or attack models.

To solve this problem, we divide a complex protocol into several less complex construction steps. Each step specifies precisely what is assumed and what is achieved. Following the tradition of constructive cryptography ${ }^{4}(\mathrm{CC})$ [16]17, we model guarantees and expectations as resources that provide a specified service to each party. Every party possesses an interface to the resources via which it can request that service. We consider resources with three interfaces, labeled $A$ (for Alice), B (for Bob) and E (for Eve). The construction notion of CC provides the following compositional guarantee: a constructed resource can be used in any other construction as an assumed resource. We obtain modularity in the sense that the overall security follows automatically from individual security proofs.

The approach has already been applied successfully in many other contexts. For example, the results in [56] shed new light on the definitions of public-key encryption schemes and even led to a new security definition.

\section{Preliminaries}

Notation. We describe our systems with pseudocode using the following conventions: We write $x \leftarrow y$ for assigning the value $y$ to the variable $x$. For a

\footnotetext{
${ }^{4}$ We suspect that alternative definitional frameworks, like treating ASCs in the UC framework [4] or RSIM [121], would yield closely related findings.
} 


\begin{tabular}{l}
$\frac{\operatorname{Real}_{\Pi}}{\text { Initialization }}$ \\
\hline$K \leftarrow \mathcal{K}$ \\
$\frac{\text { Oracle Enc }}{\text { Input: }(N, A, M) \in \mathcal{N} \times \mathcal{A} \times \mathcal{M}}$ \\
$\quad C \leftarrow \mathcal{E}(K, N, A, M)$ \\
$\quad$ return $C$ \\
$\frac{\text { Oracle } \operatorname{Dec}}{\text { Input: }(N, A, C) \in \mathcal{N} \times \mathcal{A} \times \mathcal{C}}$ \\
$M \leftarrow \mathcal{D}(K, N, A, C)$ \\
$\quad$ return $M$ \\
\hline
\end{tabular}

\section{Ideal $_{\Pi}$}

\begin{tabular}{l}
$\overline{\text { Initialization }}$ \\
\hline$K \nleftarrow \mathcal{K}$ \\
\hline Oracle Enc \\
\hline Input: $(N, A, M) \in \mathcal{N} \times \mathcal{A} \times \mathcal{M}$ \\
$\quad C^{\prime} \leftarrow \mathcal{E}(K, N, A, M)$ \\
$C \leftarrow \Sigma^{\left|C^{\prime}\right|}$ \\
$\quad$ return $C$ \\
\hline Oracle Dec \\
Input: $(N, A, C) \in \mathcal{N} \times \mathcal{A} \times \mathcal{C}$ \\
$\quad$ return $\perp$
\end{tabular}

Fig. 1. Real and ideal security game for AEAD-schemes.

distribution $\mathcal{D}$ over some set, $x \nleftarrow \mathcal{D}$ denotes sampling $x$ according to $\mathcal{D}$. For a finite set $X, x \leftarrow X$ denotes assigning to $x$ a uniformly random value in $X$. Typically queries to systems consist of a suggestive keyword and a list of arguments (e.g., (send, $M$ ) to send the message $M$ ). We ignore keywords in writing the domains of arguments, e.g., (send, $M) \in \mathcal{M}$ indicates that $M \in \mathcal{M}$.

AEAD. Let $\Sigma$ be an alphabet (a finite nonempty set). Typically an element of $\Sigma$ is a bit $(\Sigma=\{0,1\})$ or a byte $\left(\Sigma=\{0,1\}^{8}\right)$. For a string $x \in \Sigma^{*},|x|$ denotes its length. We define the syntax of a scheme for authenticated encryption with associated data (AEAD) following [22].

Definition 1. An $A E A D$-scheme $\Pi$ is a triple of algorithms $\Pi=(\mathcal{K}, \mathcal{E}, \mathcal{D})$, where $\mathcal{K}$ is a randomized algorithm that samples a key $K \in \Sigma^{*}, \mathcal{E}$ is a deterministic algorithm that maps a key $K \in \Sigma^{*}$, a nonce $N \in \mathcal{N}$, additional data $A \in \mathcal{A}$, and a message $M \in \mathcal{M}$ to a ciphertext $C \in \mathcal{C}$, and $\mathcal{D}$ is a deterministic algorithm that maps $(K, N, A, C) \in \Sigma^{*} \times \mathcal{N} \times \mathcal{A} \times \mathcal{C}$ to $\mathcal{M} \cup\{\perp\}$. We assume the domains $\mathcal{N}, \mathcal{A}, \mathcal{M}$, and $\mathcal{C}$ are equal to $\Sigma^{*}$ and require for all $K, N, A, M \in \Sigma^{*}$ that $\mathcal{D}(K, N, A, \mathcal{E}(K, N, A, M))=M$. We further require the length of a ciphertext $|\mathcal{E}(K, N, A, M)|$ only depend on the length of the corresponding message $|M|$.

We define the security game for AEAD-schemes using the all-in-one formulation from [10]. A scheme is considered secure if all valid and efficient adversaries $\mathcal{A}$ have poor advantage according to the following definition.

Definition 2. We define the advantage of an adversary $\mathcal{A}$ as the difference in the probability that it outputs 1 in the real and ideal games defined in Fig. 1.

$$
\operatorname{Adv}_{\Pi}^{\mathrm{ae}}(\mathcal{A}):=\operatorname{Pr}\left[\mathcal{A}^{\text {Real }_{\Pi}}=1\right]-\operatorname{Pr}\left[\mathcal{A}^{\text {Ideal }_{\Pi}}=1\right]
$$

An adversary is valid if it does not repeat Enc or Dec queries, does not ask queries $\operatorname{Enc}(N, A, M)$ and $\operatorname{Enc}\left(N, A^{\prime}, M^{\prime}\right)$ (i.e., does not repeat nonces), and does not ask a query $\operatorname{Dec}(N, A, C)$ where $C$ was returned by a preceding query $\operatorname{Enc}(N, A, M)$. 


\section{Revisiting the Functionality and Modeling of Communication Channels}

In constructive cryptography, communication channels are modeled as resources with three interfaces: Interface A for sender Alice, interface B for receiver Bob, and interface $E$ for adversary Eve. Different types of such channels have been studied that differ in the capabilities of the adversary Eve [6]16[18].

In the following paragraphs, we present a formalization of secure and insecure channels and argue why in many applications users might need more services than provided by either. To resolve this mismatch, we introduce a new type of channel, which we call augmented secure channel $(A S C)$, that provides those missing functionalities.

\subsection{Existing Formalizations}

Insecure channel. The insecure channel IC allows messages to be input repeatedly at interface A. Each message is subsequently leaked at the E-interface. At interface $E$, arbitrary messages (including those that were previously input at interface A) can be injected such that they are delivered to B. This channel does not give any security guarantees to Alice and Bob. A formal description is provided in Fig. 2.

\section{Resource IC}

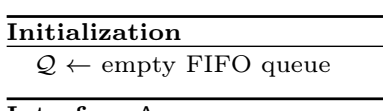

Interface A

Input: (send, $M) \in \Sigma^{*}$

$\mathcal{Q}$.enqueue $(M)$

output $M$ at interface $\mathrm{E}$

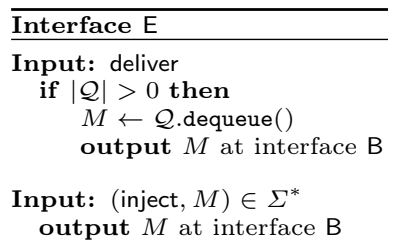

Fig. 2. The insecure channel resource.

Secure channel. The typical formalization of a secure channel follows the same basic structure as an insecure channel but where the ability of the adversary is limited to seeing the length of the transmitted messages and to deliver messages input at interface A. In particular, the adversary cannot inject new messages or induce out-of-order message delivery. A description of the secure channel can be derived from Fig. 2 by omitting the inject-query and by restricting the leakage at interface $\mathrm{E}$ to $|M|$ on inputs (send, $M$ ) at interface $\mathrm{A}$. 


\subsection{What Service Should a Secure Channel Provide?}

In many relevant security protocols, like TLS, transmitted data packets are usually divided into a header part and a payload part. While both are required to be authentic, only the payload has to remain confidential.

We further observe that the header often contains context information since binding a message to a given context is good security-engineering practice. Moreover, parts of the context are already known to the receiver. This part does not have to be transmitted but should still be authenticated. This suggests splitting the header into two parts: an explicit part and an implicit part that describe the unknown and known parts of the header, respectively.

We conclude that there is a need for an abstract functionality that allows one to transmit a message together with the explicit part of a header such that the message remains private and the message as well as both the explicit and the implicit part of the header are authenticated.

Augmented secure channel. We now present the channel abstraction that formalizes the desired service. The augmented secure channel ASC is described in Fig. 3 The sender can provide a triple consisting of the explicit part of a header $E \in \mathcal{H}_{\mathrm{E}}$, the implicit part of the header $I \in \mathcal{H}_{\mathrm{I}}$, and a message $M \in \mathcal{M}$. The message remains confidential and the explicit part of the header is leaked at the adversarial interface. If the receiver knows the implicit part of the header, he can recover the message using the query (fetch, $I$ ) and verify the authenticity of the message and both parts of the header. If the verification fails, the system stops delivering messages and signals an error by outputting $\perp$. The adversary has the ability to deliver messages and to inject a special element that will terminate the channel at the receiver's side once fetched. Delivering a message notifies the receiver of the new message and provides him with the explicit part of the header.

\section{Constructing an Augmented Secure Channel via Authenticated Encryption}

After motivating the need for the new channel ASC, we now show how to construct it using an AEAD-scheme. We first introduce the assumed resources from which we construct ASC and describe the protocol that achieves this construction. We finally prove the security of our construction.

\subsection{Assumed Resources}

We construct the augmented secure channel from an insecure channel IC and a shared secret key. We introduce a shared key resource $\mathbf{S K}_{\mathcal{K}}$ for some key distribution $\mathcal{K}$ that initially chooses a key according to $\mathcal{K}$ and on input getKey at interface $A$ or $B$, outputs this key at the corresponding interface; interface $E$ remains inactive. See Fig. 4 for pseudocode. We denote by $\left[\mathbf{S K}_{\mathcal{K}}, \mathbf{I C}\right]$ the resource that provides at each interface access to the corresponding interface of both the 


\section{Resource ASC}

Initialization

$\mathcal{S} \leftarrow$ empty FIFO queue

$\mathcal{R} \leftarrow$ empty FIFO queue

halt $\leftarrow 0$

Interface A

Input: (send, $E, I, M) \in \mathcal{H}_{\mathrm{E}} \times \mathcal{H}_{\mathrm{I}} \times \mathcal{M}$ $\mathcal{S}$.enqueue $((E, I, M))$

output $(E,|M|)$ at interface $\mathrm{E}$

Interface B

Input: (fetch, $I) \in \mathcal{H}_{\mathrm{I}}$

if $|\mathcal{R}|>0$ and halt $=0$ then

$\left(E^{\prime}, I^{\prime}, M^{\prime}\right) \leftarrow \mathcal{R}$.dequeue ()

if $I^{\prime}=I \neq \perp$ then

else

output $M^{\prime}$ at interface B

halt $\leftarrow 1$

output $\perp$ at interface $B$

Fig. 3. Description of ASC, an augmented secure channel.

key and the channel. The resource $\mathbf{S K}_{\mathcal{K}}$ can be constructed by some key exchange protocol, e.g., during the TLS handshake.

\section{Resource $\mathrm{SK}_{\mathcal{K}}$}

\begin{tabular}{ll}
\hline $\begin{array}{l}\text { Initialization } \\
k \leftarrow \mathcal{K}\end{array}$ & \\
$\begin{array}{l}\text { Interface } \mathrm{A} \\
\begin{array}{c}\text { Input: getKey } \\
\text { output } k \text { at } \mathrm{A} .\end{array}\end{array}$ & $\begin{array}{c}\text { Input: getKey } \\
\text { output } k \text { at } \mathrm{B} .\end{array}$
\end{tabular}

Fig. 4. The shared secret key resource.

\subsection{Protocol}

A protocol is modeled in constructive cryptography as a pair of converters that specify the actions of both honest parties Alice and Bob. A converter is a system with two interfaces: the inner interface in is connected to an interface of a resource and the outer interface out becomes the new connection point of that resource towards the environment. Attaching a converter to an interface changes the local behavior at that interface and hence yields a new resource ${ }^{5}$

\footnotetext{
${ }^{5}$ For example, the resource $\mathrm{enc}_{\Pi}{ }^{\mathrm{A}} \mathrm{dec}_{\Pi}{ }^{\mathrm{B}}\left[\mathbf{S} \mathbf{K}_{\mathcal{K}}, \mathbf{I C}\right]$ is obtained by attaching Alice's converter enc ${ }_{\Pi}$ at interface $A$ and Bob's converter $\operatorname{dec}_{\Pi}$ at interface $B$ of $\left[\mathbf{S K}_{\mathcal{K}}, \mathbf{I C}\right]$, where the interfaces are indicated by superscripts.
} 
For an AEAD-scheme $\Pi=(\mathcal{K}, \mathcal{E}, \mathcal{D})$, we present the protocol $\left(\mathrm{enc}_{\Pi}, \operatorname{dec}_{\Pi}\right)$ as pseudocode in Fig. 5. The converter for the sender, enc ${ }_{\Pi}$, accepts inputs of the form (send, $E, I, M$ ) at its outer interface and encrypts the message $M$ using $\mathcal{E}$. The nonce is implemented as a counter, the additional date ${ }^{6}$ is $A=(E, I)$ and the key is provided by the key-resource $\mathbf{S K}_{\mathcal{K}}$. An encoding of the resulting ciphertext and the explicit part of the header is output to the insecure channel IC.

The receiver converter $\operatorname{dec}_{\Pi}$ receives inputs from IC and queues the headerciphertext pairs internally in a queue $\mathcal{Q}$. For each newly arrived message a notification is output at the outer interface. The next ciphertext $C$ in the queue is decrypted if $\operatorname{dec}_{\Pi}$ is invoked with the implicit part of the corresponding header. The parameters for decryption are again the header as the additional data, the counter as the nonce and the shared key. On success, the corresponding plaintext is output at the outer interface. If decryption fails, the converter stops and signals an error by outputting $\perp$.

\section{Converter enc $\Pi$}

\section{Initialization}

$N \leftarrow 0$

output getKey to $\mathrm{SK}_{K}$

let $K$ be returned value from $\mathbf{S K}_{\mathcal{K}}$

$$
\begin{aligned}
& \text { Interface out } \\
& \text { Input: (send, } E, I, M) \in \mathcal{H}_{\mathrm{E}} \times \mathcal{H}_{\mathrm{I}} \times \mathcal{M} \\
& A \leftarrow(E, I) \\
& C \leftarrow \mathcal{E}(K, N, A, M) \\
& N \leftarrow N+1 \\
& \quad \text { output (send, }(E, C)) \text { to IC }
\end{aligned}
$$

\section{Converter $\operatorname{dec}_{\Pi}$}

Initialization

$\mathcal{Q} \leftarrow$ empty FIFO queue

$N \leftarrow 0$

halt $\leftarrow 0$

output getKey to $\mathbf{S K}_{\mathcal{K}}$

let $K$ be returned value from $\mathbf{S K}_{\mathcal{K}}$

Interface in

Input: $(E, C) \in \mathcal{H}_{\mathrm{E}} \times \mathcal{C}$ from IC

if halt $=0$ then

$\mathcal{Q}$.enqueue $((E, C))$

output (newMsg, $E$ ) at out

Interface out

Input: (fetch, $I) \in \mathcal{H}_{\mathrm{I}}$

if $|\mathcal{Q}|>0$ and halt $=0$ then

$(E, C) \leftarrow \mathcal{Q}$.dequeue ()

$A \leftarrow(E, I)$

$M \leftarrow \mathcal{D}(K, N, A, C)$

$N \leftarrow N+1$

if $M=\perp$ then halt $\leftarrow 1$

else output $M$ at out

Fig. 5. The protocol converters for the sender (left) and the receiver (right) that construct $\mathbf{A S C}$ via an $\mathrm{AEAD}$-scheme $\Pi=(\mathcal{K}, \mathcal{E}, \mathcal{D})$.

\subsection{The Construction Notion}

In order to show that the protocol $\left(\mathrm{enc}_{\Pi}, \operatorname{dec}_{\Pi}\right)$ constructs $\mathbf{A S C}$ from $\left[\mathbf{S K} \mathbf{K}_{\mathcal{K}}, \mathbf{I C}\right]$ in the sense of constructive cryptography, we have to prove the availability

\footnotetext{
$\overline{{ }^{6} \text { Here, }(E, I)} \in \mathcal{H}_{\mathrm{E}} \times \mathcal{H}_{\mathrm{I}}$ denotes an encoding of that pair as an element in $\mathcal{A}$. Abusing notation, we generally do not distinguish between a tuple and its encoding as an element in $\Sigma^{*}$.
} 
condition and the security condition that are derived from the general construction notion in [16].

Random experiments. Both conditions make statements about random experiments DR in which a distinguisher $\mathbf{D}$ plays the role of an interactive environment for some resource $\mathbf{R}$. The distinguisher $\mathbf{D}$ is a system that provides inputs to the connected resource and receives the outputs generated by the resource. For example, $\mathbf{D}\left(\mathrm{enc}_{\Pi}{ }^{\mathrm{A}} \mathrm{dec}_{\Pi}{ }^{\mathrm{B}}\left[\mathbf{S} \mathbf{K}_{\mathcal{K}}, \mathbf{I C}\right]\right)$ is the experiment that captures "the protocol in action" in the environment provided by $\mathbf{D}$. More concretely, in each step of these experiments, the distinguisher provides an input to one of the interfaces $A$, $B$, or $E$ and observes the output that is generated in reaction to that input. This process continues iteratively by having $\mathbf{D}$ providing adaptively the next input and receiving the next output. The experiment ends by $\mathbf{D}$ outputting a bit 0 or 1 that indicates its guess to which system it is connected. The distinguishing advantage of $\mathbf{D}$ for two resources $\mathbf{R}$ and $\mathbf{S}$ is defined as

$$
\Delta^{\mathbf{D}}(\mathbf{R}, \mathbf{S})=\operatorname{Pr}[\mathbf{D R}=1]-\operatorname{Pr}[\mathbf{D S}=1] .
$$

Availability condition. The first condition captures the situation when no attacker interferes with the protocol execution. We require that in this case, the intended functionality is available to the honest parties. This condition can be seen as a correctness condition for the protocol.

No attacker being present is formalized by a special converter dlv that is attached at interface $\mathrm{E}$ and always ensures the delivery of messages. Concretely, on any input at its inner interface, dlv outputs deliver to the channel connected to its inner interface and does not provide any service at its outer interface. Formally, the availability condition places a bound on the advantage in distinguishing the systems enc ${ }_{\Pi}{ }^{\mathrm{A}} \operatorname{dec}_{\Pi}{ }^{\mathrm{B}} \operatorname{dlv}^{\mathrm{E}}\left[\mathbf{S K} \mathcal{K}_{\mathcal{K}}, \mathbf{I C}\right]$ and $\operatorname{dlv}^{\mathrm{E}} \mathbf{A S C}$, i.e., a bound on

$$
\begin{aligned}
& \Delta^{\mathbf{D}}\left(\operatorname{enc}_{\Pi}{ }^{\mathrm{A}} \operatorname{dec}_{\Pi}{ }^{\mathrm{B}} \mathrm{dlv}^{\mathrm{E}}\left[\mathbf{S K}_{\mathcal{K}}, \mathbf{I C}\right], \mathrm{dlv}^{\mathrm{E}} \mathbf{A S C}\right) \\
& \quad=\operatorname{Pr}\left[\mathbf{D}\left(\mathrm{enc}_{\Pi}{ }^{\mathrm{A}} \operatorname{dec}_{\Pi}^{\mathrm{B}} \mathrm{dlv}^{\mathrm{E}}\left[\mathbf{S} \mathbf{K}_{\mathcal{K}}, \mathbf{I C}\right]\right)=1\right]-\operatorname{Pr}\left[\mathbf{D}\left(\operatorname{dlv}^{\mathrm{E}} \mathbf{A S C}\right)=1\right]
\end{aligned}
$$

for any distinguisher $\mathbf{D}$.

Security condition. The second condition captures the situation where an adversary attacks the protocol execution using its capabilities at interface $\mathrm{E}$. The effects of such an attack have to be indistinguishable from the effects in the system corresponding to the constructed resource with some simulator attached at the adversarial interface. This captures that all attacks on the protocol can be translated by a simulator to an attack on the constructed resource. Turned around, if the constructed resource is secure by definition, there is no successful attack on the protocol. More concretely, the security condition places a bound on the 
advantage in distinguishing the systems enc ${ }_{\Pi}{ }^{\mathrm{A}} \operatorname{dec}_{\Pi}{ }^{\mathrm{B}}\left[\mathbf{S K}_{\mathcal{K}}, \mathbf{I C}\right]$ and $\operatorname{sim}_{\mathrm{ASC}}^{\mathrm{E}} \mathbf{A S C}$ for some simulator $\operatorname{sim}_{\mathrm{ASC}}$, i.e., a bound on

$$
\begin{aligned}
& \Delta^{\mathbf{D}}\left(\operatorname{enc}_{\Pi}{ }^{\mathrm{A}} \operatorname{dec}_{\Pi}^{\mathrm{B}}\left[\mathbf{S K} \mathbf{K}_{\mathcal{K}}, \mathbf{I C}\right], \operatorname{sim}_{\mathrm{ASC}}^{\mathrm{E}} \mathbf{A S C}\right) \\
& \quad=\operatorname{Pr}\left[\mathbf{D}\left(\operatorname{enc}_{\Pi}^{\mathrm{A}} \operatorname{dec}_{\Pi}^{\mathrm{B}}\left[\mathbf{S K} \boldsymbol{K}_{\mathcal{K}}, \mathbf{I C}\right]\right)=1\right]-\operatorname{Pr}\left[\mathbf{D}\left(\operatorname{sim}_{\mathrm{ASC}}^{\mathrm{E}} \mathbf{A S C}\right)=1\right]
\end{aligned}
$$

for any distinguisher $\mathbf{D}$.

\subsection{Proof of the Construction}

The following two lemmata relate the AEAD-security game to the distinguishing advantage in the availability and security condition, respectively. Note that the availability condition does not follow directly from the correctness of the AEAD-scheme. This is because it is not excluded that a ciphertext gets decrypted to some message $M \neq \perp$ if the wrong additional data is supplied, while the system $\mathrm{dlv}^{\mathrm{E}}$ ASC always returns $\perp$ if the wrong value for $I$ is input at interface $B$. We need the security of the AEAD-scheme to conclude that such invalid decryptions can only occur with small probability.

Lemma 1. There is an (efficient) transformation $\rho$ described in the proof that maps distinguishers $\mathbf{D}$ for two resources to valid adversaries $\mathcal{A}=\rho(\mathbf{D})$ for the $A E A D$-security game such that

$$
\Delta^{\mathbf{D}}\left(\operatorname{enc}_{\Pi}{ }^{\mathrm{A}} \operatorname{dec}_{\Pi}^{\mathrm{B}} \mathrm{dlv}^{\mathrm{E}}\left[\mathbf{S K} \mathcal{K}_{\mathcal{K}}, \mathbf{I C}\right], \operatorname{dlv}^{\mathrm{E}} \mathbf{A S C}\right) \leq \mathbf{A d v}_{\Pi}^{\mathrm{ae}}(\rho(\mathbf{D})) .
$$

Proof. In enc $_{\Pi}{ }^{\mathrm{A}} \operatorname{dec}_{\Pi}{ }^{\mathrm{B}} \mathrm{dlv}^{\mathrm{E}}\left[\mathbf{S} \mathbf{K}_{\mathcal{K}}, \mathbf{I C}\right]$, the converter dlv is attached at interface $\mathrm{E}$ and answers any output produced by IC with the input deliver. This essentially converts IC into a reliable transmission channel: whatever pair $(E, C)$ is input by converter $\mathrm{enc}_{\Pi}$, it is immediately delivered to $\operatorname{dec}_{\Pi}$ that outputs a notification (newMsg, $E$ ) at its outer interface. Furthermore, if the $i$ th input at interface $\mathrm{A}$ is (send, $E_{i}, I_{i}, M_{i}$ ), and the $i$ th input at interface $\mathrm{B}$ is (fetch, $I_{i}$ ), then the output at interface B is $M_{i}$. The same holds for system $\mathrm{dlv}^{\mathrm{E}} \mathbf{A S C}$. Only if the $i$ th input at interface $\mathrm{B}$ is (fetch, $I_{i}^{\prime}$ ) for $I_{i}^{\prime} \neq I_{i}$, then the behavior of the two systems can differ: While $\mathrm{dlv}^{\mathrm{E}}$ ASC always returns $\perp$ in this case, for $\operatorname{enc}_{\Pi}{ }^{\mathrm{A}} \operatorname{dec}_{\Pi}{ }^{\mathrm{B}} \mathrm{dlv}^{\mathrm{E}}\left[\mathbf{S K}_{\mathcal{K}}, \mathbf{I C}\right]$ it is possible that a message $M \neq \perp$ is returned. Since this is the only difference between the two systems, we can upper bound the distinguishing advantage by the probability that $\mathbf{D}$ can provoke such an output at interface $\mathrm{B}$ when interacting with enc $_{\Pi}{ }^{\mathrm{A}} \operatorname{dec}_{\Pi}{ }^{\mathrm{B}} \mathrm{dlv}^{\mathrm{E}}\left[\mathbf{S K}_{\mathcal{K}}, \mathbf{I C}\right]$. It remains to bound the probability of this event, subsequently denoted by $\mathcal{F}$.

Note that $\mathcal{F}$ occurs exactly if the decryption algorithm of the AEAD-scheme returns a message $M \neq \perp$ on input a different additional data than used for encryption. Based on this observation, we build an adversary $\mathcal{A}$ that emulates a view towards distinguisher $\mathbf{D}$ that is identical to an interaction of $\mathbf{D}$ with enc $_{\Pi}{ }^{\mathrm{A}} \operatorname{dec}_{\Pi}{ }^{\mathrm{B}} \mathrm{dlv}^{\mathrm{E}}\left[\mathbf{S} \mathbf{K}_{\mathcal{K}}, \mathbf{I C}\right]$ if $\mathcal{A}$ gets access to its real oracles. The probability of provoking event $\mathcal{F}$ is preserved in this case. In contrast, if $\mathcal{A}$ gets access to the 
ideal oracles, the condition for event $\mathcal{F}$ cannot be satisfied as we argue below. This is a suitable distinguishing criterion.

More formally, the reduction $\rho$ is defined as follows: The adversary $\mathcal{A}=\rho(\mathbf{D})$ initially sets $N_{A}, N_{B} \leftarrow 0$, initializes an empty FIFO queue $\mathcal{Q}$, and then emulates an execution to $\mathbf{D}$ as follows. When $\mathbf{D}$ inputs (send, $E, I, M$ ) at interface $\mathbf{A}$, $\mathcal{A}$ ask the query $\left(N_{A},(I, E), M\right)$ to the oracle Enc to receive the answer $C$. It then executes $N_{A} \leftarrow N_{A}+1$ and $\mathcal{Q}$.enqueue $((E, I, M, C))$, and emulates the output (newMsg, $E$ ) at interface B for $\mathbf{D}$. Inputs (fetch, $I^{\prime}$ ) at interface B are ignored if $\mathcal{Q}$ is empty. Otherwise, $\mathcal{A}$ executes $(E, I, M, C) \leftarrow \mathcal{Q}$.dequeue( $)$. If $I^{\prime}=I$, it sets $M^{\prime}=M$; if $I^{\prime} \neq I$, it asks the query $\left(N_{B},\left(I^{\prime}, E\right), C\right)$ to the oracle Dec to receive the answer $M^{\prime}$. It then sets $N_{B} \leftarrow N_{B}+1$ and emulates the output $M^{\prime}$ at interface B for $\mathbf{D}$.

If $M^{\prime} \neq \perp$ and $I^{\prime} \neq I$ (i.e., the event $\mathcal{F}$ occurs), $\mathcal{A}$ stops and returns 1 . If $M^{\prime}=\perp, \mathcal{A}$ ignores subsequent inputs at interface $\mathbf{B}$. When $\mathbf{D}$ outputs a bit and $\mathcal{F}$ has not occurred, $\mathcal{A}$ returns 0 . Observe that if $\mathcal{A}$ gets access to the ideal oracles, the conditions of event $\mathcal{F}$ cannot be met. We conclude the proof by noting that $\mathcal{A}$ is a valid adversary and $\mathbf{A d v}_{I I}^{\text {ae }}(\rho(\mathbf{D}))$ equals the probability of the event $\mathcal{F}$.

The next lemma implies the security condition of the construction:

Lemma 2. For the simulator $\operatorname{sim}_{\mathrm{ASC}}$ defined in Fig. 6, there is an (efficient) transformation $\rho^{\prime}$ described in the proof that maps distinguishers $\mathbf{D}$ for two resources to valid adversaries $\mathcal{A}=\rho^{\prime}(\mathbf{D})$ for the AEAD-security game such that

$$
\Delta^{\mathbf{D}}\left(\operatorname{enc}_{\Pi}^{\mathrm{A}} \operatorname{dec}_{\Pi}^{\mathrm{B}}\left[\mathbf{S} K_{\mathcal{K}}, \mathbf{I C}\right], \operatorname{sim}_{\mathrm{ASC}}^{\mathrm{E}} \mathbf{A S C}\right) \leq \mathbf{A d v}_{\Pi}^{\mathrm{ae}}\left(\rho^{\prime}(\mathbf{D})\right) .
$$

\section{Converter $\operatorname{sim}_{\mathrm{ASC}}$}

\begin{tabular}{l}
$\frac{\overline{\text { Initialization }}}{\mathcal{Q}_{1}, \mathcal{Q}_{2} \leftarrow \text { empty FIFO queues }}$ \\
let $\hat{K} \in \mathcal{K}, \hat{N} \in \mathcal{N}, \hat{A} \in \mathcal{A}$ be arbitrary \\
\hline Interface in \\
Input: $(E, \ell) \in \mathcal{H}_{\mathrm{E}} \times \mathbb{N}$ \\
choose $M_{\ell} \in \mathcal{M}$ with $\left|M_{\ell}\right|=\ell$ \\
$C_{\ell} \leftarrow \mathcal{E}\left(\hat{K}, \hat{N}, \hat{A}, M_{\ell}\right)$ \\
$C \leftarrow \Sigma^{\left|C_{\ell}\right|}$ \\
$\mathcal{Q}_{1}$.enqueue $((E, C))$ \\
$\mathcal{Q}_{2}$.enqueue $((E, C))$ \\
output $(E, C)$ at out
\end{tabular}

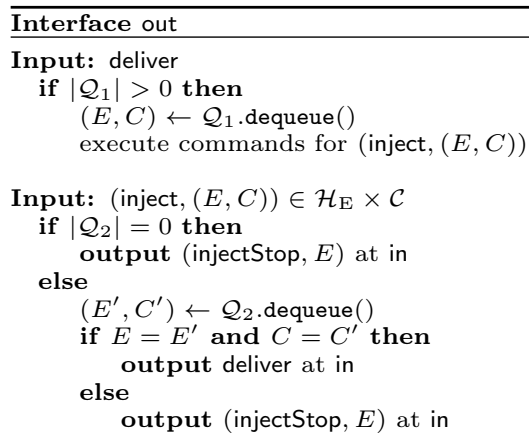

Fig. 6. The simulator for the security condition of the construction of ASC. 
Proof. Let $\mathbf{D}$ be a distinguisher for $\mathrm{enc}_{\Pi}{ }^{\mathrm{A}} \operatorname{dec}_{\Pi}^{\mathrm{B}}\left[\mathbf{S K}_{\mathcal{K}}, \mathbf{I C}\right]$ and $\operatorname{sim}_{\mathrm{ASC}}^{\mathrm{E}} \mathbf{A S C}$. We define an adversary $\mathcal{A}=\rho^{\prime}(\mathbf{D})$ for the AEAD-security game as follows. The adversary $\mathcal{A}$ initially sets $N_{A}, N_{B}$, flag $\leftarrow 0$, initializes an empty FIFO queue $\mathcal{S}$ and two empty list: $\mathbf{7}^{7} \mathcal{L}$ and $\mathcal{R}$, and then emulates an execution of $\mathbf{D}$ by translating inputs of the distinguisher to oracle queries as well as answers from the oracles to outputs of the resource for $\mathbf{D}$. There are four types of inputs $\mathbf{D}$ can make:

(send, $E, I, M)$ at interface A: If $\mathcal{R}$ contains strictly less than $N_{A}+1$ elements, $\mathcal{A}$ asks the query $\left(N_{A},(E, I), M\right)$ to the oracle Enc and receives the answer $C$. It then stores $\left(N_{A}, E, I, M, C\right)$ in the list $\mathcal{L}$, emulates the output $(E, C)$ at interface $\mathbf{E}$ for $\mathbf{D}$, sets $N_{A} \leftarrow N_{A}+1$, and executes $\mathcal{S}$.enqueue $((E, C))$.

If $\mathcal{R}$ contains at least $N_{A}+1$ elements, there is a pair $\mathcal{R}\left[N_{A}\right]=(E, C) . \mathcal{A}$ asks the query $\left(N_{A},(E, I), C\right)$ to the oracle Dec to receive the plaintext $M$. If $M \neq \perp, \mathcal{A}$ sets flag $\leftarrow 1$, returns 1 as its decision and halts. If $M=\perp$, the tuple $\left(N_{A}, E, I, \perp, C\right)$ is stored in $\mathcal{L}$ and $\mathcal{A}$ asks the query $\left(N_{A},(E, I), M\right)$ to the oracle Enc, receives the answer $C$ and stores $\left(N_{A}, E, I, M, C\right)$ in the list $\mathcal{L}$. Finally, $\mathcal{A}$ emulates the output $(E, C)$ at interface $\mathrm{E}$ for $\mathbf{D}$, sets $N_{A} \leftarrow N_{A}+1$, and executes $\mathcal{S}$.enqueue $((E, C))$.

deliver at interface $\mathrm{E}$ : If $|\mathcal{S}|>0, \mathcal{A}$ executes $(E, C) \leftarrow \mathcal{S}$.dequeue () followed by $\mathcal{R} \leftarrow \mathcal{R} \|(E, C)$. If $\perp$ has not been output at interface $\mathrm{B}, \mathcal{A}$ emulates the output (newMsg, $E$ ) at interface B.

(inject, $(E, C)$ ) at interface $\mathrm{E}$ : The adversary $\mathcal{A}$ executes $\mathcal{R} \leftarrow \mathcal{R} \|(E, C)$. If $\perp$ has not been output at interface $\mathrm{B}, \mathcal{A}$ emulates the output (newMsg, $E$ ) at interface $B$.

(fetch, $I$ ) at interface $\mathrm{B}$ : If $\mathcal{R}$ is empty, the input is ignored. Otherwise, $\mathcal{A}$ executes $(E, C) \leftarrow \mathcal{R}\left[N_{B}\right]$. If $\left(N_{B}, E, I, \perp, C\right)$ is in $\mathcal{L}, \mathcal{A}$ emulates the output $\perp$ at interface $\mathrm{B}$ and ignores subsequent inputs at interface $\mathrm{B}$. If $\left(N_{B}, E, I, M, C\right)$ is in $\mathcal{L}$ for some $M \in \mathcal{M}, \mathcal{A}$ emulates the output $M$ at interface $\mathrm{B}$ for $\mathbf{D}$ and sets $N_{B} \leftarrow N_{B}+1$. Otherwise, $\mathcal{A}$ asks the query $\left(N_{B},(E, I), C\right)$ to the oracle Dec to receive the plaintext $M$. The output $M$ is emulated at interface $\mathrm{B}$ and the counter $N_{B}$ is incremented. If $M=\perp, \mathcal{A}$ ignores subsequent inputs at interface $B$.

When $\mathbf{D}$ outputs a bit $b$ and if $\mathrm{flag}=0, \mathcal{A}$ returns $b$ and halts. Note that $\mathcal{A}$ is a valid adversary since it asks at most one Enc and Dec query for each nonce (and therefore does not repeat queries) and never asks a query to the oracle Dec for a ciphertext that has been returned by a query to Enc for the same parameters (because for such ciphertext, the corresponding tuple is in the list $\mathcal{L}$ ). To analyze the success probability of $\mathcal{A}$, let $F$ be the random variable that takes on the value of $f$ lag at the end of the random experiment between $\mathcal{A}$ and $\mathbf{R e a l}_{\Pi}$.

We claim that the view of $\mathbf{D}$ when connected to $\operatorname{sim}_{\mathrm{ASC}}^{\mathrm{E}} \mathbf{A S C}$ is identical to the view emulated by $\mathcal{A}$ with access to the ideal oracles. Additionally, the view of $\mathbf{D}$ when connected to $\mathrm{enc}_{\Pi}{ }^{\mathrm{A}} \operatorname{dec}_{\Pi}^{\mathrm{B}}\left[\mathbf{S} \mathbf{K}_{\mathcal{K}}, \mathbf{I C}\right]$ is identical to the view emulated by

\footnotetext{
${ }^{7}$ For a list $L$, we denote by $L \| x$ the list $L$ with $x$ appended. Furthermore, the $i$ th element of a list $L$ with $n$ elements is denoted by $L[i]$ for $i \in\{0, \ldots, n-1\}$.
} 
$\mathcal{A}$ with access to the real oracles as long as $f l a g=0$. This implies the statement of the lemma:

$$
\begin{aligned}
& \operatorname{Adv}_{\Pi}^{\text {ae }}(\mathcal{A})=\operatorname{Pr}\left[\mathcal{A}^{\text {Real }_{\Pi}}=1\right]-\operatorname{Pr}\left[\mathcal{A}^{\text {Ideal }_{\Pi}}=1\right] \\
& =\operatorname{Pr}[F=1]+\operatorname{Pr}[F=0] \cdot \operatorname{Pr}\left[\mathcal{A}^{\text {Real }_{\Pi}}=1 \mid F=0\right]-\operatorname{Pr}\left[\mathcal{A}^{\text {Ideal }_{\Pi}}=1\right] \\
& =\operatorname{Pr}\left[\mathbf{D}\left(\operatorname{enc}_{\Pi}^{\mathrm{A}} \operatorname{dec}_{\Pi}^{\mathrm{B}}\left[\mathbf{S K}_{\mathcal{K}}, \mathbf{I C}\right]\right)=1\right] \\
& \geq \operatorname{Pr}\left[\mathbf{D}\left(\operatorname{enc}_{\Pi}{ }^{\mathrm{A}} \operatorname{dec}_{\Pi}{ }^{\mathrm{B}}\left[\mathbf{S K}_{\mathcal{K}}, \mathbf{I C}\right]\right)=1\right]-\operatorname{Pr}\left[\mathbf{D}\left(\operatorname{sim}_{\mathrm{ASC}}^{\mathrm{E}} \mathbf{A S C}\right)=1\right] \\
& =\Delta^{\mathbf{D}}\left(\operatorname{enc}_{\Pi}{ }^{\mathrm{A}} \operatorname{dec}_{\Pi}^{\mathrm{B}}\left[\mathbf{S K}_{\mathcal{K}}, \mathbf{I C}\right], \operatorname{sim}_{\mathrm{ASC}}^{\mathrm{E}} \mathbf{A S C}\right) \text {. }
\end{aligned}
$$

To prove this claim, we distinguish the possible inputs by $\mathbf{D}$ and compare the resulting outputs:

(send, $E, I, M)$ at interface A: In system enc ${ }_{\Pi}^{\mathrm{A}} \operatorname{dec}_{\Pi}^{\mathrm{B}}\left[\mathbf{S} \mathbf{K}_{\mathcal{K}}, \mathbf{I C}\right]$, the converter enc $_{\Pi}$ evaluates $C \leftarrow \mathcal{E}(K, N,(E, I), M)$, where $N$ is the number of sent messages before this input. The explicit part $E$ of the header is sent together with $C$ over IC, which outputs the pair $(E, C)$ at interface $\mathrm{E}$. The same output is emulated by $\mathcal{A}$ in the real game since the oracle Enc in this case also evaluates the algorithm $\mathcal{E}$.

In the system $\operatorname{sim}_{\mathrm{ASC}}^{\mathrm{E}} \mathbf{A S C}$, the triple $(E, I, M)$ is inserted into the senders queue of ASC and the pair $(E,|M|)$ is output to the simulator $\operatorname{sim}_{\mathrm{ASC}}$, which in turn generates a uniformly random ciphertext $C$ of the same length as ciphertexts for $M$. Note that by Definition 1 , the length of ciphertexts only depend on the length of the message, so the values of $\hat{K}, \hat{N}$, and $\hat{A}$ used by $\operatorname{sim}_{\mathrm{ASC}}$ to determine this length are irrelevant. The simulator then stores $(E, C)$ in its own queue for later reference and outputs this pair at interface $\mathrm{E}$. Note that Enc in Ideal $\Pi$ generates ciphertexts with the same distribution, so the view emulated by $\mathcal{A}$ is identical.

deliver at interface $\mathrm{E}$ : If the sender's queue it non-empty, the next element $(E, C)$ is dequeued from it and $\mathbf{D}$ receives the output (newMsg, $E$ ) from interface $B$ if there has not been an output $\perp$ in both systems and in the emulated view.

(inject, $(E, C)$ ) at interface $\mathrm{E}$ : $\mathrm{In} \mathrm{enc}_{\Pi}{ }^{\mathrm{A}} \operatorname{dec}_{\Pi}{ }^{\mathrm{B}}\left[\mathbf{S} \mathbf{K}_{\mathcal{K}}, \mathbf{I C}\right]$, the injected pair is inserted into the receiver's queue of the converter $\operatorname{dec}_{\Pi}$ and the notification (newMsg, $E$ ) is output at interface B.

In $\operatorname{sim}_{\mathrm{ASC}}^{\mathrm{E}}$ ASC, the simulator checks whether the injected pair is equal to the top-element $\left(E^{\prime}, C^{\prime}\right)$ of its queue $\mathcal{Q}_{2}$. If this is the case, the simulator outputs deliver with the effect that the notification (newMsg, $E$ ) is

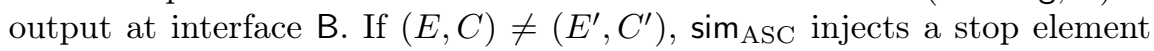
by (injectStop, $E$ ), which also yields the output (newMsg, $E$ ) at interface B. Note that by definition of ASC, this element is guaranteed to yield $\perp$ when fetched at interface B.

We see that in the emulation by $\mathcal{A}, \mathbf{D}$ receives (newMsg, $E$ ) from interface $\mathrm{B}$ if there has not been an output $\perp$ before at interface $B$. The same holds for both systems $\operatorname{enc}_{\Pi}{ }^{\mathrm{A}} \operatorname{dec}_{\Pi}^{\mathrm{B}}\left[\mathbf{S K}_{\mathcal{K}}, \mathbf{I C}\right]$ and $\operatorname{sim}_{\mathrm{ASC}}^{\mathrm{E}}$ ASC in an interaction with D. 
(fetch, $I$ ) at interface B: Assume this is the $i$ th input at interface $\mathrm{B}$, there have been at least $i$ inputs deliver or inject at interface $\mathrm{E}$, and there has not been an output $\perp$ so far (otherwise the input is always ignored). In the system enc ${ }_{\Pi}{ }^{\mathrm{A}} \operatorname{dec}_{\Pi}{ }^{\mathrm{B}}\left[\mathbf{S} \mathbf{K}_{\mathcal{K}}, \mathbf{I C}\right]$, the converter $\operatorname{dec}_{\Pi}$ retrieves the top element of its queue. This value is equal to the $i$ th delivered or injected pair $(E, C)$ at interface $\mathrm{E}$. The converter $\operatorname{dec}_{\Pi}$ then computes $M \leftarrow \mathcal{D}(K, i-1,(E, I), C)$ and outputs $M$.

In the view emulated by $\mathcal{A}$ in the real game, $(E, C)$ also corresponds to the $i$ th delivered or injected pair. If the tuple $(i-1, E, I, M, C)$ is found in $\mathcal{L}$ for some $M \in \mathcal{M}, M$ is output at interface B. By the correctness of the AEAD-scheme, $M$ is then equal to the output of the algorithm $\mathcal{D}$ for the corresponding parameters. If no such tuple is in $\mathcal{L}, \mathcal{A}$ decrypts $C$ with the corresponding parameters using the oracle Dec and also outputs the resulting message at interface B. Since the real oracle Dec evaluates $\mathcal{D}$, we conclude that the views are identical in this case.

In $\operatorname{sim}_{\mathrm{ASC}}^{\mathrm{E}} \mathbf{A S C}$, the resource checks whether $I=I^{\prime}$, where $\left(E^{\prime}, I^{\prime}, M^{\prime}\right)$ is the next element in the queue $\mathcal{R}$. If this is the case, it outputs $M^{\prime}$ at interface B, otherwise it outputs $\perp$. Furthermore, only those elements can be successfully fetched that do not correspond to stop-elements $(\perp, \perp, \perp)$. By construction of the simulator, the $i$ th element of the sender's queue is only delivered if the $i$ th injected pair $(E, C)$ at interface $\mathrm{E}$ matches the simulated pair output at interface $\mathrm{E}$ in reaction to the $i$ th input (send, $E^{\prime}, I^{\prime}, M^{\prime}$ ) at interface $\mathrm{A}$. In any other case, a stop-element is injected into the receiver's queue.

To determine whether the values of the $i$ th injection match the simulated values for the $i$ th input at interface $\mathrm{A}, \operatorname{sim}_{\mathrm{ASC}}$ maintains the queue $\mathcal{Q}_{2}$ such that its top element, after $i$ injections, stores exactly these values. Note that the queue $\mathcal{Q}_{1}$ on the other hand is only needed to simulate the queue of the insecure channel IC in the real world and to figure out the next message in the simulation of a deliver-request.

In the view emulated by $\mathcal{A}$ in the ideal game, the list $\mathcal{L}$ ensures that the same message $M^{\prime}$ is output at interface $\mathrm{B}$ if all the values match as above. Furthermore, if there is not a match, the output is $\perp$ because the ideal oracle Dec always returns $\perp$. In particular, the condition that the $i$ th simulated pair correspond to the $i$ th injected pair is equivalent to requiring that the tuple $(i-1, E, I, M, C)$, for some message $M$, is an element of $\mathcal{L}$.

Hence, the views for $\mathbf{D}$ are also identical in this case.

This concludes the proof of the claim and thus of the lemma.

The following theorem summarizes the results from Lemma 1 and Lemma 2.

Theorem 1. The protocol $\left(\mathrm{enc}_{\Pi}, \operatorname{dec}_{\Pi}\right)$ constructs ASC from $\left[\mathbf{S K}_{\mathcal{K}}, \mathbf{I C}\right]$. More specifically, we have for the simulator $\operatorname{sim}_{\mathrm{ASC}}$ in Fig. 6 and for all distinguishers $\mathbf{D}$

$$
\begin{aligned}
\Delta^{\mathrm{D}}\left(\operatorname{enc}_{\Pi}{ }^{\mathrm{A}} \operatorname{dec}_{\Pi}{ }^{\mathrm{B}} \operatorname{dlv}^{\mathrm{E}}\left[\mathbf{S K}_{\mathcal{K}}, \mathbf{I C}\right], \operatorname{dlv}^{\mathrm{E}} \mathbf{A S C}\right) & \leq \mathbf{A d v}_{\Pi}^{\mathrm{ae}}(\rho(\mathbf{D})) \\
\text { and } \quad \Delta^{\mathrm{D}}\left(\operatorname{enc}_{\Pi}{ }^{\mathrm{A}} \operatorname{dec}_{\Pi}^{\mathrm{B}}\left[\mathbf{S K}_{\mathcal{K}}, \mathbf{I C}\right], \operatorname{sim}_{\mathrm{ASC}}^{\mathrm{E}} \mathbf{A S C}\right) & \leq \mathbf{A d v}_{\Pi}^{\mathrm{ae}}\left(\rho^{\prime}(\mathbf{D})\right),
\end{aligned}
$$


where $\rho$ and $\rho^{\prime}$ are the reductions defined in the proofs of Lemma 1 and Lemma 2 . respectively.

\section{The Goal of the TLS 1.3 Record Layer}

Version 1.3 of TLS is currently in draft state at the IETF 8 Unlike TLS 1.2, the new version of the record payload protection protocol mandates AEAD ciphers 9 and the format of the authenticated data has changed. More specifically, in the current draft, the authenticated data in TLS 1.3 consists of the sequence number of the current fragment, the protocol version, and the message type, e.g., whether it is an "alert," a "handshake," or an "application" message. The nonce of the AEAD cipher is chosen in a specific way based on the sequence number ${ }^{10}$ This section treats the record protocol and assumes that a shared key has been derived in the TLS handshake.

\subsection{Formalizing the Goal of TLS Record Payload Protection}

What does it mean for the TLS record layer to be secure? We propose a simple answer to this question in the form of a new channel abstraction. Each packet in the TLS record layer contains a payload and specifies its associated type. While the entire packet is authenticated, only the content of the packet has to be private and hidden from the attacker. This resembles a specific type of channel: a secure channel where messages are tagged with a non-private type-flag from the set of types $\mathcal{T}:=\{0, \ldots, 255\}$. The TLS record payload protection can be considered secure if it provably constructs this secure channel. We formalize this channel as the resource $\mathbf{S E C}_{\mathrm{TLS}}$ and provide a formal description thereof in Fig. $7{ }^{11}$

Note that in contrast to ASC, the channel $\mathbf{S E C}_{\mathrm{TLS}}$ does not contain an implicit part of the header and messages are directly delivered to Bob without the need to fetch them. Therefore, $\mathbf{S E C}_{\mathrm{TLS}}$ does not allow the authentication of data without sending it. One can thus view $\mathbf{S E C}$ TLS as an augmented secure channel that is more restricted than ASC but also simpler to use.

\subsection{Achieving the Goal}

In this section, we present a construction of the channel $\mathbf{S E C}_{\mathrm{TLS}}$ from ASC. To this end, we introduce the protocol (tlsSnd, tlsRcv), which is described in Fig. 8

\footnotetext{
${ }^{8}$ We refer to the most recent draft (retrieved on August 28, 2015) that is available for download at https://tools.ietf.org/html/draft-ietf-tls-tls13-08.

9 Previous versions of TLS supported MAC-then-Encrypt modes.

${ }^{10}$ Until draft 5, which was the reference for an earlier version of this paper, the choice of the nonce was not specified, and it was transmitted together with the ciphertext.

${ }^{11}$ While applications usually provide data to TLS as a sequence of multi-byte strings, TLS only guarantees that the same stream of bytes, as the concatenation of the individual strings, is delivered. TLS does not guarantee that the boundaries between the multi-byte strings are preserved as chosen by the application, cf. [7. The message $M$ in Fig. 7 is to be understood as the multi-byte string used within the TLS protocol, which is not necessarily the same as chosen by the higher-level application.
} 


\section{Resource SEC $\mathrm{TLS}$}

\begin{tabular}{l}
\hline $\begin{array}{l}\mathcal{S} \leftarrow \text { empty FIFO queue } \\
\text { halt } \leftarrow 0\end{array}$ \\
\hline Interface A \\
Input: $($ send, $T, M) \in \mathcal{T} \times \mathcal{M}$ \\
$\mathcal{S}$.enqueue $((T, M))$ \\
output $(T,|M|)$ at interface $\mathrm{E}$
\end{tabular}

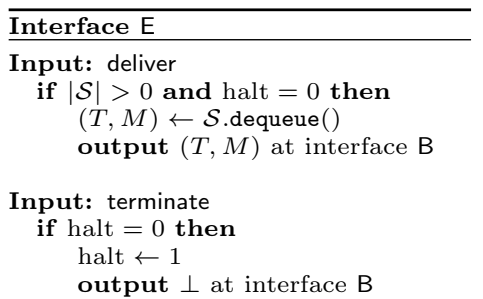

Fig. 7. Description of the channel SEC $_{\text {TLS }}$.

and manages the usage of the resource ASC. We adhere closely to the current TLS specification in setting the corresponding values. The implicit part $I$ of the header consists of the protocol version $V$ (which corresponds to $\{3,4\}$ and consists of two bytes ${ }^{12}$ and the explicit part $E$ consists of the message type $T$ (one byte).

\section{Converter tlsSind}

\begin{tabular}{l}
\hline$\frac{\text { Initialization }}{V \leftarrow\{3,4\}}$ \\
\hline Interface out \\
Input: (send, $T, M) \in \mathcal{T} \times \mathcal{M}$ \\
output (send, $T, V, M)$ to ASC
\end{tabular}

\section{Converter tlsRcv}

\begin{tabular}{l}
\hline$\frac{\text { Initialization }}{V \leftarrow\{3,4\}}$ \\
\hline Interface in \\
\hline Input: (newMsg, $T) \in \mathcal{T}$ \\
if halt $=0$ then \\
output (fetch, $V$ ) to ASC \\
let $M$ be returned value from ASC \\
if $M \neq \perp$ then \\
output $(T, M)$ at out \\
else \\
halt $\leftarrow 1$ \\
output $\perp$ at out
\end{tabular}

Fig. 8. The protocol converters for the sender (left) and the receiver (right) that construct $\mathbf{S E C}_{\mathrm{TLS}}$ from ASC.

Theorem 2. The protocol (tlsSnd, tlsRev) constructs $\mathbf{S E C}_{\mathrm{TLS}}$ from ASC. More specifically, we have for the simulator $\operatorname{sim}_{\mathrm{TLS}}$ defined in Fig. 9 and for all distinguishers $\mathbf{D}$

$$
\begin{aligned}
\Delta^{\mathrm{D}}\left(\mathrm{tlsSnd}^{\mathrm{A}} \mathrm{tlsRcv}^{\mathrm{B}} \mathrm{dlv}^{\mathrm{E}} \mathbf{A S C}, \mathrm{dlv}^{\mathrm{E}} \mathbf{S E C}_{\mathrm{TLS}}\right) & =0 \\
\text { and } \quad \Delta^{\mathrm{D}}\left(\mathrm{tlsSnd}^{\mathrm{A}} \mathrm{tlsRcv}^{\mathrm{B}} \mathbf{A S C}, \operatorname{sim}_{\mathrm{TLS}}^{\mathrm{E}} \mathbf{S E C}_{\mathrm{TLS}}\right) & =0 .
\end{aligned}
$$

\footnotetext{
$\overline{12}$ The value $\{3,4\}$ corresponds to TLS version 1.3 . The reason for this value is that
} the version of TLS 1.0, as the successor of SSL 3.0, is encoded as the value $\{3,1\}$. 


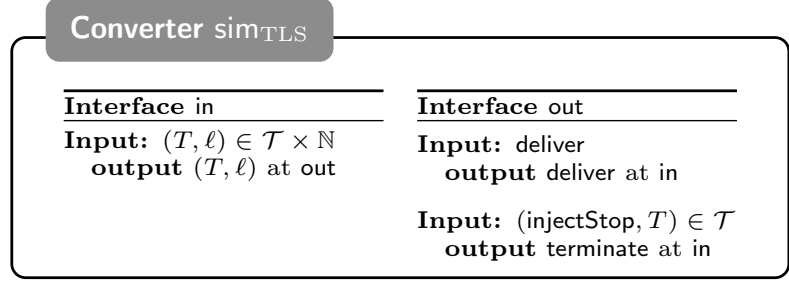

Fig. 9. The simulator for the security condition of the construction of $\mathbf{S E C}_{\mathrm{TLS}}$.

Proof. The availability condition (1) is easy to verify: On input (send, $T, M$ ) at interface $\mathrm{A}$, the system $\mathrm{dlv}^{\mathrm{E}} \mathbf{S E} \mathbf{C}_{\mathrm{TLS}}$ directly outputs $(T, M)$ at interface $\mathrm{B}$. The same holds for system tlsSnd ${ }^{A}$ llsRcv $^{B} \operatorname{dlv}^{E}$ ASC: On input (send, $T, M$ ), the converter tlsSnd inputs (send, $T, V, M$ ) to ASC. The converter tlsRcv then obtains the notification (newMsg, $T$ ) and queries (fetch, $V$ ) to ASC, which results in the output $M$ from ASC, which in turn triggers tlsRcv to output $(T, M)$. Since the two systems behave identically, every distinguisher has advantage 0 in distinguishing them, i.e., (1) follows.

To verify the security condition (2), we distinguish the possible inputs to the system:

Input (send, $T, M)$ at interface A: In the system tlsSnd ${ }^{\mathrm{A}} \mathrm{tlsRcv}^{\mathrm{B}} \mathbf{A S C}$, this input results in the converter tlsSnd inputting (send, $T, V, M$ ) to ASC, which yields the output $(T,|M|)$ at interface $\mathbf{E}$ of $\mathbf{A S C}$. In $\operatorname{sim}_{\mathrm{TLS}}^{\mathrm{E}} \mathbf{S E C}_{\mathrm{TLS}}$, the values $(T,|M|)$ are given to the simulator, which then outputs $(T,|M|)$ at its outer interface.

Input deliver at interface $\mathrm{E}$ : In $\mathrm{tlsSnd}^{\mathrm{A}} \mathrm{tlsRcv}^{\mathrm{B}} \mathrm{ASC}$, if the queue $\mathcal{S}$ in $\mathbf{A S C}$ is empty, nothing happens. Otherwise, the converter tlsRcv receives the notification (newMsg, T). Then, tlsRcv inputs (fetch, $V$ ) to ASC if it has not already halted. In this case, there have been only inputs deliver at interface $\mathrm{E}$ and therefore the verification within ASC succeeds. Thus, tlsRcv obtains the message $M$ and outputs $(T, M)$.

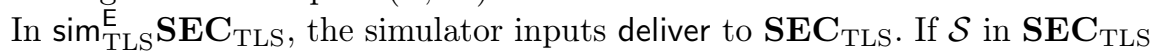
is empty, nothing happens. Otherwise, the next tuple $(T, M)$ in $\mathcal{S}$ is output at interface $B$ if the channel has not halted before.

Input (injectStop, $T$ ) at interface $\mathrm{E}$ : In the system $\mathrm{tlsSnd}^{\mathrm{A}} \mathrm{tlsRcv}^{\mathrm{B}} \mathbf{A S C}$, the notification (newMsg, $T$ ) is output to tlsRcv. The converter tlsRcv then outputs (fetch, $V$ ) to ASC and since the element is an inserted empty element, the verification within ASC fails and tlsRcv outputs $\perp$ and stops by setting halt $\leftarrow 1$.

In $\operatorname{sim}_{\mathrm{TLS}}^{\mathrm{E}} \mathbf{S E C}_{\mathrm{TLS}}, \operatorname{sim}_{\mathrm{TLS}}$ terminates the session, which causes the output $\perp$ at interface $B$ and results in no further messages being processed by Bob.

To see that the two described systems behave identically, we only have to observe that they both terminate the session if an empty message is injected into the channel and that all inputs are delivered in order until termination. We again 
conclude that every distinguisher has advantage 0 in distinguishing these systems, i.e., we obtain (2). This completes the proof.

\subsection{Using the Protocol in TLS 1.3}

We have shown that (tlsSnd, tlsRcv) constructs $\mathbf{S E C}_{\mathrm{TLS}}$ from ASC. Since by Theorem 1 , the protocol $\left(\mathrm{enc}_{\Pi}, \operatorname{dec}_{\Pi}\right)$ constructs the channel ASC from a shared secret key and an insecure channel, we can invoke the composition theorem of constructive cryptography to conclude that the composition of both protocols constructs $\mathbf{S E C} \mathbf{C L S}_{\mathrm{TLS}}$ from a shared key $\mathbf{S K}_{\mathcal{K}}$ and an insecure channel IC. See Fig. 10 for a graphical illustration of the composed protocol $\left(\mathrm{tls}_{\mathrm{A}}, \mathrm{t} \mathrm{s}_{\mathrm{B}}\right)$.

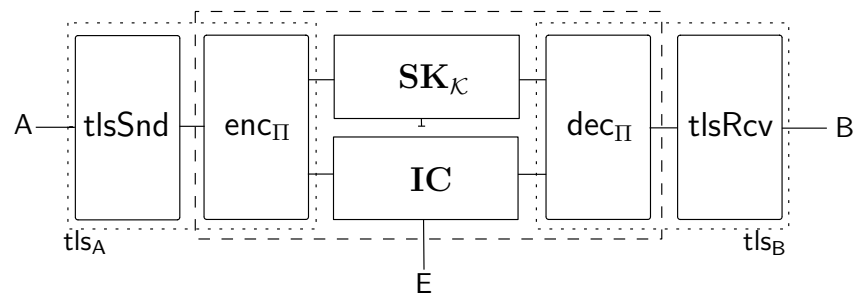

Fig. 10. Illustration of the composed protocol $\left(\mathrm{tls}_{\mathrm{A}}, \mathrm{t} \mathrm{s}_{\mathrm{B}}\right)$. For a secure AEAD-scheme, the resource $\mathrm{enc}_{\Pi}{ }^{\mathrm{A}} \operatorname{dec}_{\Pi}{ }^{\mathrm{B}}\left[\mathbf{S K}_{\mathcal{K}}, \mathbf{I C}\right]$ inside the dashed box in the center is indistinguishable from $\operatorname{sim}_{\mathrm{ASC}}^{\mathrm{E}} \mathrm{ASC}$.

The protocol for the sender tls $\mathrm{s}_{\mathrm{A}}$ works as follows: On input (send, $T, M$ ), the message $M$ is encrypted with a call to the AEAD-scheme as $C \leftarrow \mathcal{E}(K, N, A, M)$, where $K$ is the shared key retrieved from $\mathbf{S K}_{\mathcal{K}}, N$ is the internal counter and $A=(T, V)$ is the additional data. Finally, the pair $(T, C)$ is sent over the insecure channel.

The protocol for the receiver $\mathrm{tl}_{\mathrm{B}}$ works analogously: On input a new pair $(T, C)$ from IC, the ciphertext is decrypted to $M \leftarrow \mathcal{D}(K, N, A, C)$, where $N$ is the internal counter, $A$ is the additional data, and $K$ is the shared key as above. Note that the implicit part of the header is fixed and provided by tIsRcv immediately after receiving the notification (newMsg, $T$ ) from $\operatorname{dec}_{\Pi}$.

In summary, the protocol $\left(\mathrm{t}_{\mathrm{s}}, \mathrm{t} \mathrm{ls}_{\mathrm{B}}\right)$ provably achieves the goal of the TLS record layer. Note that the key resource $\mathbf{S K}_{\mathcal{K}}$ is constructed by the handshake protocol if both parties are authenticated. In [14, the authors consider the more general case where only one party is authenticated, which yields a weaker key resource. We have chosen the setting where both sides are authenticated to simplify the presentation, but we point out that our result can be generalized to the more general setting straightforwardly.

Our proposal. Our results shown in this paper suggest minor modifications to the current draft of TLS 1.3: 
1. The nonce of the AEAD scheme can be set to the counter value left-padded with zeros to be of the appropriate length.

2. The sequence number can be removed from the additional data part.

3. After the handshake, the version number does not need to be transmitted explicitly as part of the TLS record. However, it should still be part of the additional data.

Our proposal comes with a rigorous security proof, which guarantees that our choice of parameters is adequate. For example, this clarifies that the nonce need not be unpredictable or derived from other values, which is a priori unclear.

We are aware that item 3 would require a new structure of TLS fragments and hence there might be objections against this change. However, we stress that only respecting item 1 and item 2 of our proposal is also secure (i.e., constructs $\left.\mathbf{S E C}_{\mathrm{TLS}}\right)$. The proof for the case where the version number is moved to the explicit part of the header is essentially identical to the one presented in Sect. 5.2.

Acknowledgments. Ueli Maurer was supported by the Swiss National Science Foundation (SNF), project no. 200020-132794. Björn Tackmann was supported by the Swiss National Science Foundation (SNF) via Fellowship no. P2EZP2_155566 and the NSF grants CNS-1116800 and CNS-1228890. Much of the work on this paper was done while Phil Rogaway was visiting Ueli Maurer's group at ETH Zurich. Many thanks to Ueli for hosting that sabbatical. Rogaway was also supported by NSF grants CNS-1228828 and CNS-1314885.

\section{References}

1. Backes, M., Pfitzmann, B., Waidner, M.: The reactive simulatability (RSIM) framework for asynchronous systems. Inf. Comput. 205(12), 1685-1720 (Dec 2007)

2. Bellare, M., Namprempre, C.: Authenticated encryption: Relations among notions and analysis of the generic composition paradigm. In: Advances in Cryptology ASIACRYPT 2000, pp. 531-545. Springer (2000)

3. Bellare, M., Rogaway, P.: Encode-then-encipher encryption: How to exploit nonces or redundancy in plaintexts for efficient cryptography. In: Advances in Cryptology ASIACRYPT 2000, pp. 317-330. Springer (2000)

4. Canetti, R.: Universally composable security: a new paradigm for cryptographic protocols. In: 42nd IEEE Symposium on Foundations of Computer Science, pp. 136-145. IEEE (2001)

5. Canetti, R., Krawczyk, H., Nielsen, J.B.: Relaxing chosen-ciphertext security. In: Advances in Cryptology - CRYPTO 2003, pp. 565-582. Springer (2003)

6. Coretti, S., Maurer, U., Tackmann, B.: Constructing confidential channels from authenticated channels - public-key encryption revisited. In: Advances in Cryptology - ASIACRYPT 2013, Part I, pp. 134-153. Springer (2013)

7. Fischlin, M., Günther, F., Marson, G. A., Paterson, K. G.: Data Is a Stream: Security of Stream-Based Channels. In Advances in Cryptology - CRYPTO 2015, pp. 545-564, Springer (2015)

8. Gajek, S., Manulis, M., Pereira, O., Sadeghi, A., Schwenk, J.: Universally composable security analysis of TLS. In: Proceedings of ProvSec 2008, pp. 313-327 (2008) 
9. He, C., Sundararajan, M., Datta, A., Derek, A., Mitchell, J.: A modular correctness proof of IEEE 802.11i and TLS. In: Proceedings of the ACM Conference on Computer and Communications Security (ACM CCS'05), pp. 2-15 (2005)

10. Hoang, V.T., Krovetz, T., Rogaway, P.: Robust authenticated-encryption: AEZ and the problem that it solves. In: Advances in Cryptology - EUROCRYPT 2015, pp. 15-44. Springer (2015)

11. Jager, T., Kohlar, F., Schäge, S., Schwenk, J.: On the Security of TLS-DHE in the Standard Model. In: Advances in Cryptology - CRYPTO 2012, pp. 273-293. Springer (2012)

12. Jutla, C.: Encryption modes with almost free message integrity. In: Advances in Cryptology - EUROCRYPT 2001, pp. 529-544. Springer (2001)

13. Katz, J., Yung, M.: Unforgeable encryption and chosen ciphertext secure modes of operation. In: Fast Software Encryption, pp. 284-299. Springer (2001)

14. Kohlweiss, M., Maurer, U., Onete, C., Tackmann, B., Venturi, D.: (De-)Constructing TLS. Cryptology ePrint Archive, Report 2014/020 (2014)

15. Krawczyk, H., Paterson, K., Wee, H.: On the security of the TLS protocol: A systematic analysis. In: Proceedings of CRYPTO 2013, pp. 429-448 (2013)

16. Maurer, U.: Constructive cryptography - a new paradigm for security definitions and proofs. In: Mödersheim, S., Palamidessi, C. (eds.) Theory of Security and Applications, Lecture Notes in Computer Science, vol. 6993, pp. 33-56. Springer (2012)

17. Maurer, U., Renner, R.: Abstract cryptography. In: Chazelle, B. (ed.) The Second Symposium on Innovations in Computer Science, ICS 2011, pp. 1-21. Tsinghua University Press (2011)

18. Maurer, U., Rüedlinger, A., Tackmann, B.: Confidentiality and integrity: a constructive perspective. In: TCC 2012, pp. 209-229. Springer (2012)

19. Morrissey, P., Smart, N., Warinschi, B.: A modular security analysis of the TLS handshake protocol. In: Proceedings of ASIACRYPT 2008, pp. 55-73 (2008)

20. Paterson, K, Ristenpart, T., Shrimpton, T.: Tag Size Does Matter: Attacks and Proofs for the TLS Record Protocol. In: Advances in Cryptology - ASIACRYPT 2011, pp. 372-389 (2011)

21. Pfitzmann, B., Waidner, M.: A model for asynchronous reactive systems and its application to secure message transmission. In: Proceedings of the 2001 IEEE Symposium on Security and Privacy, pp. 184-200. IEEE Computer Society (2001)

22. Rogaway, P.: Authenticated-encryption with associated-data. In: Proceedings of the 9th ACM Conference on Computer and Communications Security, pp. 98-107. ACM (2002)

23. Rogaway, P., Bellare, M., Black, J.: OCB: A block-cipher mode of operation for efficient authenticated encryption. ACM Transactions on Information and System Security (TISSEC) 6(3), 365-403 (2003)

24. Rogaway, P., Shrimpton, T.: A provable-security treatment of the key-wrap problem. In: Advances in Cryptology - EUROCRYPT 2006, pp. 373-390. Springer (2006)

25. Wagner D., Schneier, B.: Analysis of the SSL 3.0 protocol. In: USENIX - Workshop on Electronic Commerce, pp. 29-40 (1996) 